UCRL- 101431

PREPRINT

\title{
Flashlamp Pumping of Nd:Glass Disk Amplifiers
}

H. T. Powe11, A. C. Erlandson,

K. S. Jancaitis, and J. E. Murray

This paper was prepared for submittal to

The International Congress

on Optical Science and Engineering

The Hague, Netherlands

March 13, 1990

June 12, 1990

This is a preprint of a paper intended for publication in a journal or proceedings. Since changes may be made before publication, this preprint is made available with the understanding that it will not be cited or reproduced without the permission of the author.

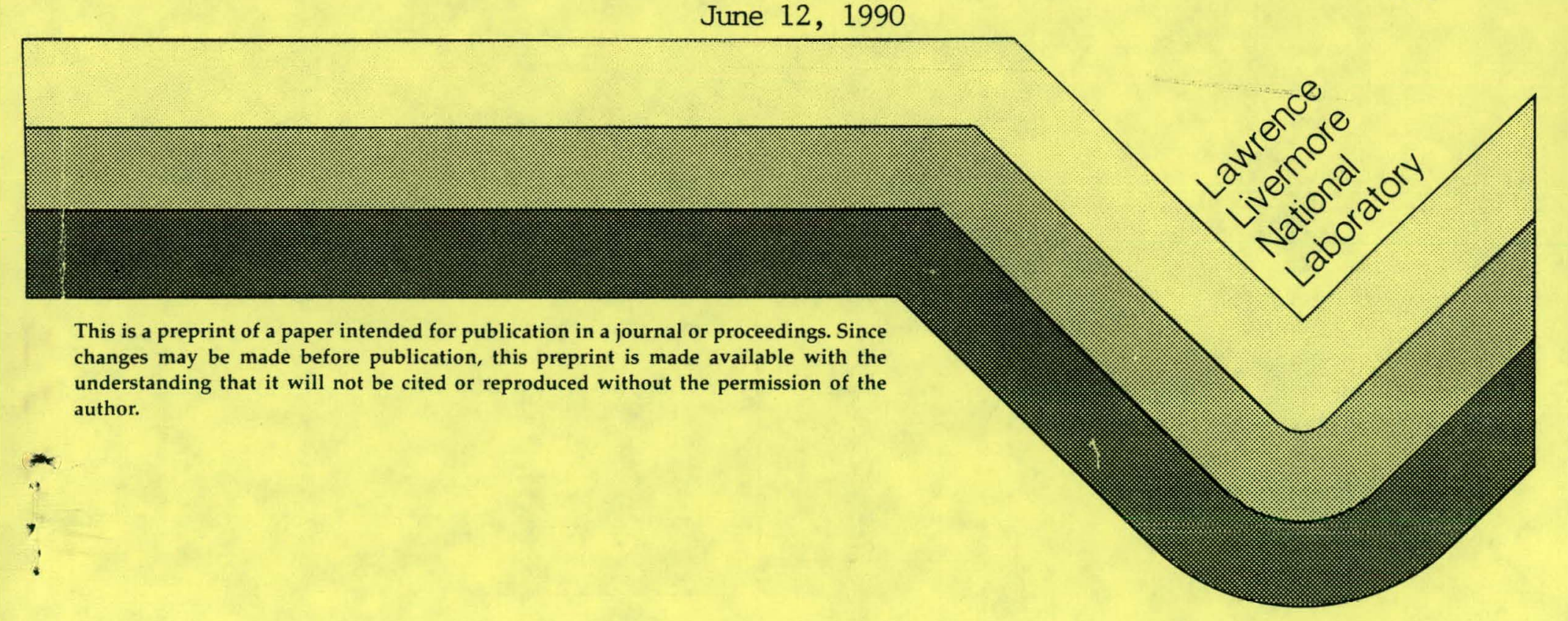




\section{DISCLAIMER}

This report was prepared as an account of work sponsored by an agency of the United States Government. Neither the United States Government nor any agency Thereof, nor any of their employees, makes any warranty, express or implied, or assumes any legal liability or responsibility for the accuracy, completeness, or usefulness of any information, apparatus, product, or process disclosed, or represents that its use would not infringe privately owned rights. Reference herein to any specific commercial product, process, or service by trade name, trademark, manufacturer, or otherwise does not necessarily constitute or imply its endorsement, recommendation, or favoring by the United States Government or any agency thereof. The views and opinions of authors expressed herein do not necessarily state or reflect those of the United States Government or any agency thereof. 


\section{DISCLAIMER}

Portions of this document may be illegible in electronic image products. Images are produced from the best available original document. 
DISCLAIMER

This document was prepared as an account of work sponsored by an agency of the United States Government. Neither the United States Government nor the University of California nor any of their employees, makes any warranty, express or implied, or assumes any legal liability or responsibility for the accuracy, completeness, or usefulness of any information, apparatus, product, or process disclosed, or represents that its use would not infringe privately owned rights. Reference herein to any specific commercial products, process, or service by trade name, trademark, manufacturer, or otherwise, does not necessarily constitute or imply its endorsement, recommendation, or favoring by the United States Government or the University of California. The views and opinions of authors expressed herein do not necessarily state or reflect those of the United States Government or the University of California, and shall not be used for advertising or product endorsement purposes. 


\title{
Flashlamp Pumping of Nd:Glass Disk Amplifiers*
}

\author{
H. T. Powell, A. C. Erlandson, K. S. Jancaitis, and J. E. Murray \\ University of California \\ Lawrence Livermore National Laboratory \\ P.O. Box 5508, L-490 \\ Livermore, CA 94550 \\ (415) $422-6149$ \\ UCRL- -101431

\section{ABSTRACT} \\ DE9 0013482
}

We present experimental results and a model of Nd:glass disk amplifiers which are used in inertial confinement fusion research. We first review our previous measurements on pulsed xenon flashlamps. We then discuss out measurements on the enhancement of the Nd fluorescence decay rate in laser disks by amplified spontaneous emission. Using these data, we have constructed a model of flashlamp pumping which treats the transfer efficiency of pump light from the flashlamps to the disks as an empirical function. We have found a simple description of this cavity transfer function which provides an excellent fit to the amplifier results for various pump pulselengths. We discuss the concept of the pump area ratio for describing the flashlamp packing density and show that amplifier performance is optimized for values of this parameter near unity. We finally present results for both a singlesegment and a multisegment disk amplifier. We have used these devices to investigate new amplifier designs for a large scale fusion driver.

\section{INTRODUCTION}

To reach ignition conditions, a driver for inertial confinement fusion (ICF) must have an output of order one megajoule at an appropriate wavelength (presumably UV) and with a carefully tailored pulseshape extending over a few nanoseconds. ${ }^{1}$ Flashlamp-pumped, harmonically-converted Nd:glass lasers are the leading candidate for meeting those requirements in the near future. The largest scale realization of flashlamp-pumped Nd:glass laser technology at present is the Nova facility at Lawrence Livermore National Laboratory. Nova has recently delivered a ten-beam output of more than $120 \mathrm{~kJ}$ at its fundamental 1.05$\mu \mathrm{m}$ wavelength and more than $50 \mathrm{~kJ}$ at its third harmonic at $0.35 \mu \mathrm{m} .2$ However, scaling this technology at an affordable cost to the size required for fusion ignition requires substantial improvement in our understanding and modeling of Nd:glass drivers.

The large disk amplifiers which provide most of the delivered laser energy are key components of Nd:glass ICF drivers. This paper describes our work over the past several years in obtaining the necessary data to construct a model which accurately describes these amplifiers. Much of our work is also relevant to smaller scale solid state lasers.

"Work performed under the auspieces of the U.S. Department of Energy by Lawrence Livermore National Laboratory under contract number W-7405-ENG-48. 
Our work on flashlamp pumping of Nd:glass disk amplifiers divides into three general categories: the output emission characteristics of flashlamps; the pump cavity transfer of flashlamp radiation to the disks; and finally the energy storage characteristics of the disks themselves. (We presume the amplifier is operated in the energy storage mode as appropriate for short-pulse energy extraction.). Each area has complications which must be treated to obtain an accurate pumping model. The complications we will discuss are:

- flashlamps exhibit an emission spectrum which varies in time and which does not respond instantaneously to the input power

- the cavity transfer efficiency of pump radiation also varies in time because of the timedependent opacity of the flashlamps (causing reabsorption of pump light) and of course varies with the cavity geometry and disk parameters.

- the decay rate of excited ions is increased by amplified spontaneous emission (ASE) which depends on the gain of the disk as well as its size and shape.

We first summarize our work on the flashlamp emission problem and then our efforts to quantify the effect of amplified spontaneous emission. In our current amplifier model, we treat the cavity transfer problem empirically by using a simple function which describes the instantaneous transfer efficiency. This function was selected to fit observed amplifier gain data. This approach is generally like that employed previously 3 although we now have a physical basis for the parameterization which is chosen and hence the fitting results have a physical meaning. Our model allows both interpolation and extrapolation to some degree to un-tested conditions and geometries. The model is then applied to two prototype disk amplifiers which we have built to understand amplifier scaling to larger sizes and to higher efficiency - our single-segment amplifier (SSA) 4 and our $2 \times 2$ multisegment amplifier (MSA). Both devices employ rectangular disks and have design features relevant to a large scale fusion driver. We have modeled both of these devices. The results illustrate the tradeoffs that can be made in cost-optimizing a large scale ICF driver.

\section{Flashlamp Emission Spectra}

In a previous paper 5 we described our work to characterize quantitatively the instantaneous emission spectra of pulsed Xe flashlamps over a range of input powers (5-90 $\mathrm{kW} / \mathrm{cm}^{2}$ ) and for various bore diameters $(0.5-4.2 \mathrm{~cm})$. Spectrally-detailed, angle-averaged emission spectra for quasi-stationary lamp conditions were obtained within what we call the Nd:glass pump region $(0.4$ to $1.0 \mu \mathrm{m})$. Angle-averaging is needed since the emission spectrum of flashlamps varies with viewing angle. These spectra were put on an absolute power basis by using "black-can" calorimetry and by apportioning the total emission into three broad spectral regions -- UV, pump, and IR. Detailed spectra were obtained only in the pump region.

Several significant conclusions resulted from this work. For flashlamps having clearfused-quartz envelopes, the total radiant efficiency (either instantaneous or time-integrated) is typically in the range 70 to $80 \%$ for pulselengths and input energies of interest (pulselengths $\sim 500 \mu \mathrm{s}$ and input energies $\sim 20 \%$ of the explosion limit). Efficiencies fall off somewhat at either shorter pulselengths or lower inputs although use of pulsed 
preionization has a strong effect in countering these tendencies (at least down to pulselengths $\sim 100 \mu \mathrm{s})$. We provide weak preionization $\left(\sim 0.1 \mathrm{~J} / \mathrm{cm}^{2}\right)$ in a short pulse occuring about $200 \mu \mathrm{s}$ before the main discharge, so that the preionization plasma has adequately diffused throughout the bore when the main pulse is applied.

The quasi-stationary description of the lamp output spectrum works quite well throughout the pulse duration when preionization is used, provided a small temporal correction is made to account for the radiative inertia of the plasma. This inertia causes roughly a $30-\mu \mathrm{s}$ time lag in the output power from the electrical input power. Physically this lag represents the time required for the high-temperature xenon plasma to dissipate (mostly radiatively) the energy which it contains. Thus it is more appropriate to describe the "state of the plasma" in a flashlamp by its output power rather than its input power in determining both its emission and absorption characteristics.

We illustrated this in our previous paper 5 by showing that the broad spectral fractions (UV, pump, and IR) as a function of time are described better by the instantaneous output power than the input power. In our present amplifier model we used the output power $\mathrm{P}_{\text {out }}(\mathrm{t})$ to characterize both the instantaneous flashlamp emission spectrum and the cavity transfer efficiency. The numerical value of $P_{\text {out }}(t)$ is somewhat arbitrary in that the actual output power can depend on the passive transmission of the flashlamp envelope. For example, the output power of lamps with UV-absorbing, cerium-doped-quartz envelopes is lower than those with clear-fused-quartz envelopes. By definition in our model, we chose the output $P_{\text {out }}(t)$ to be that which would be obtained with clear-fused-quartz envelopes. However, since both cerium-doped and clear-fused-quartz envelopes have the same transmission and hence the same emission spectra in the pump region, this definition is somewhat arbitrary.

The final conclusion that we reached in our flashlamp studies was that the instantaneous output spectra and radiant efficiencies of flashlamps having different bore diameters are nearly identical at quasi-stationary conditions when compared at the same input power per unit wall area of the bore. When experimentally comparing different lamps we have usually measured their radiant performance at the peak of the input power pulse (typically $600 \mu$ s $10 \%-10 \%$ pulsewidth) in order to minimize non-stationary effects. More generally, during the pulse the spectra are the same at the same output power per unit wall area. This scaling with power per unit area has not been noted previously by others who have generally assumed that electrical current density $\left(\mathrm{kA} / \mathrm{cm}^{2}\right)$, which gives the volumetric input power $\left(\mathrm{kW} / \mathrm{cm}^{3}\right)$, is the parameter of importance. 6 Indeed the output spectra of lamps having different bore diameters but operated at the same current density can be quite different. The observed scaling of the spectra with input power per unit wall area is characteristic of an optically-thick radiator. Scaling with input power per unit volume is characteristic of an optically-thin radiator. Pulsed Xe flashlamps come much closer to the optically-thick description even though for most conditions and wavelengths they are still in the gray-body regime.

Given our observations on temporal behavior and spectral scaling, one can understand why we choose to use $P_{\text {in }}(t)$ and $P_{\text {out }}(t)$ in units of $\mathrm{kW} / \mathrm{cm}^{2}$, power per unit wall area of the bore, as our fundamental lamp variables. Figures 1 and 2 summarize much of our findings on flashlamp radiant efficiency and broad spectral distributions versus these 
variables. The dual axes of $P_{\text {in }}(t)$ and $P_{\text {out }}(t)$ are shown to allow use of the curves for either time-varying or stationary conditions.

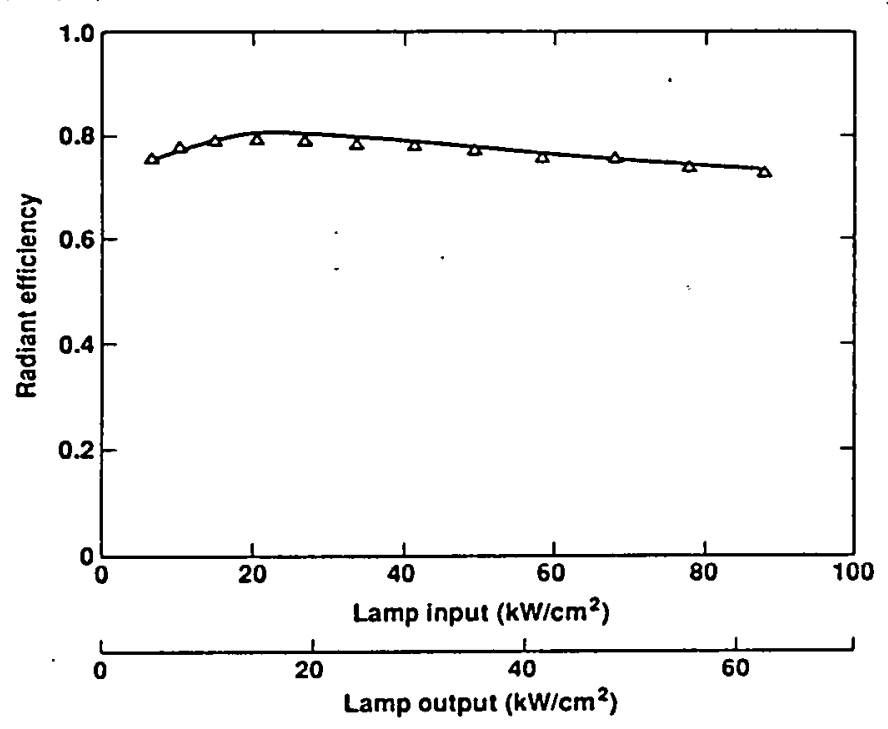

Fig. 1. Instantaneous radiant efficiency of a flashlamp as a function of both Pin and Pout. The data (triangles) and the model predictions (solid curve) are for a $1.5-\mathrm{cm}$ bore flashlamp filled to 300 Torr with Xe.

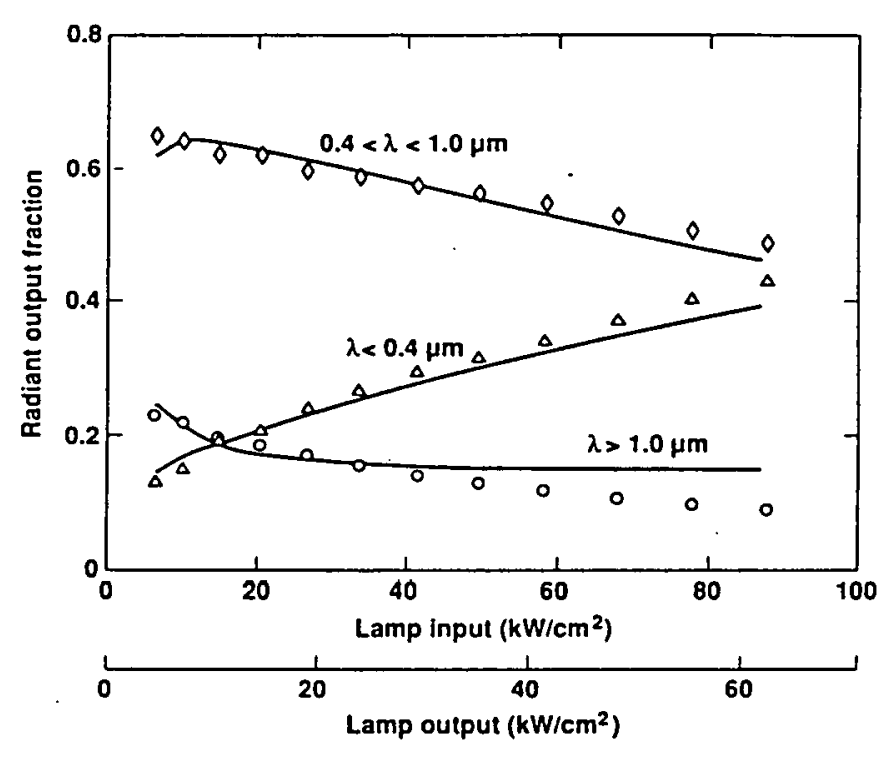

Fig. 2. UV, pump, and IR radiant output fractions as a function of both $P$ in and Pout.. The data and model predictions (solid curves) are for a $1.5-\mathrm{cm}$ bore flashlamp filled to 300 Torr with $\mathrm{Xe}$.

To facilitate our description of amplifier pumping we have reduced our flashlamp spectral measurements to a convenient empirical model which accurately fits the observed spectra. We have patterned our model on the previous work of Trenholme and Emmett. 7 Our flashlamp model assumes the instantaneous lamp emission can be described by a uniformly heated cylinder of plasma filling the lamp bore which is described by a temperature $T$ and an absorption/emission coefficient $\alpha$ which depends on wavelength. The plasma temperature and absorption coefficient depend of course on input power. According to Kirkhoff's Law these two parameters specify the output emission intensity according to

$$
\mathrm{I}(\mathrm{T}, \lambda)=\left[1-\mathrm{e}^{-\alpha(\lambda) \ell}\right] \mathrm{IBC}_{\mathrm{B}}(T, \lambda)
$$

where $\ell$ is the pathlength of plasma in the line of view and $I_{B B}$ is the Planck function. The above expression must be averaged over viewing angle to describe amplifier pumping.

Fitting our flashlamp data to this model reduces to a problem of choosing the temperature and absorption coefficient versus wavelength to match the observed spectra. We solved this problem by assuming there exists some wavelength where the lamp is opaque. At that wavelength the emission intensity is determined only by the plasma temperature. We chose the wavelength by calculating an effective blackbody temperature for our data at all wavelengths within the pump band. The highest effective temperature was then selected as the plasma temperature. The selected wavelengths were generally at 
strong Xe emission lines in the near IR. Plasma absorption coefficients were then determined from the apparent emissivity at all other wavelengths.

The temperature and absorption coefficients were determined for our range of input powers and flashlamp bore diameters and then fit to empirical formulas which are given in the Appendix. This model provides an excellent fit to the experimental data as shown by the example in Fig. 3. Figure 4 shows the absorption coefficient and emissivity which are $\checkmark$ : predicted by the model for this example.

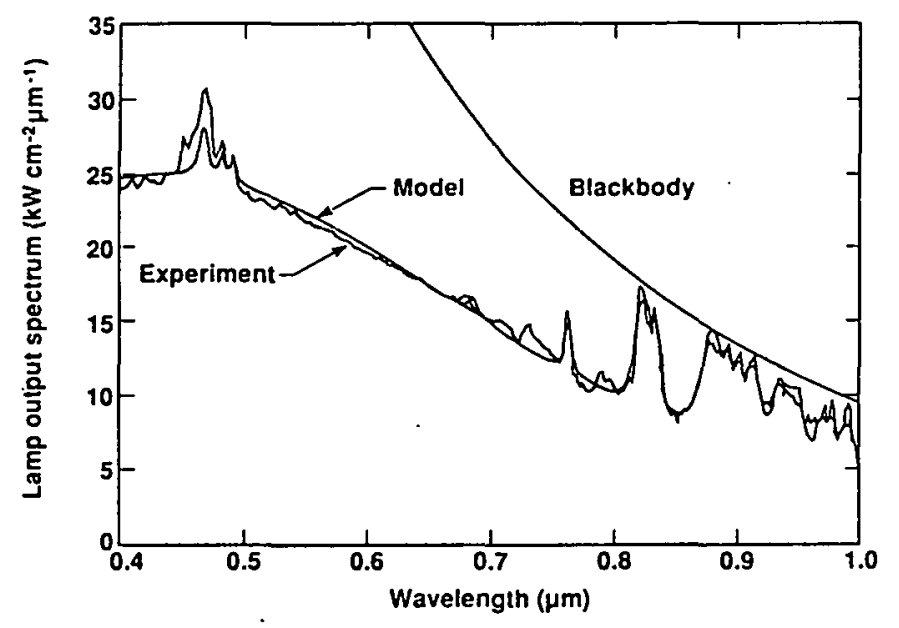

Fig. 3 Xenon flashlamp spectra (for bore diameter $=1.5 \mathrm{~cm}$, Xe fill pressure $=300$ Torr, and $P_{\text {in }}=20.1 \mathrm{~kW} / \mathrm{cm}^{2}$ ) showing excellent agreement of model predictions with measurements. The blackbody spectrum at the plasma temperature given by the model $(9200 \mathrm{~K})$ is also plotted for comparison.

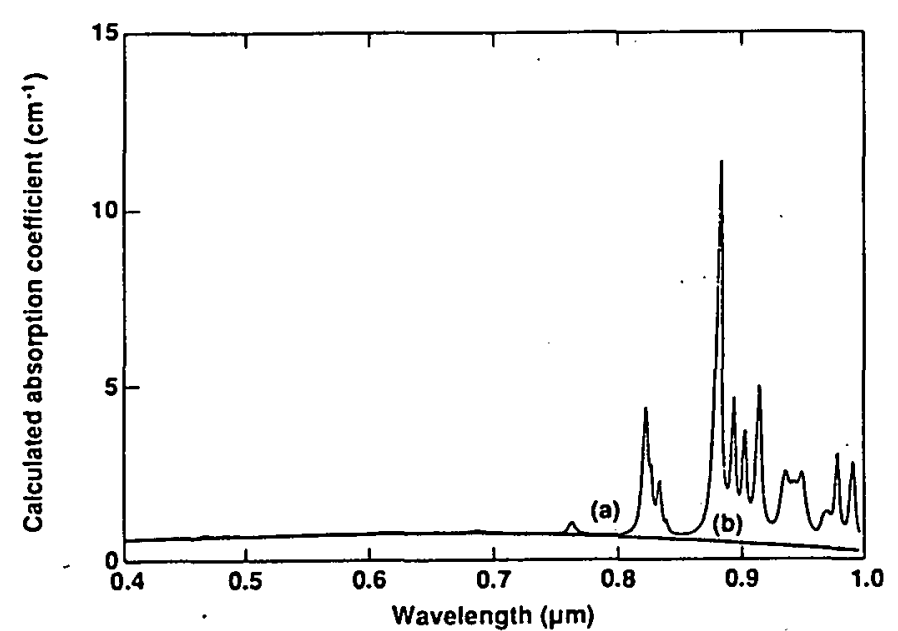

Fig. 4. Xenon flashlamp absorption coefficients predicted by the model (bore diameter $=1.5 \mathrm{~cm}, \mathrm{Xe}$ fill pressure $=300$ Torr, and $P_{\text {in }}=20.1 \mathrm{~kW} / \mathrm{cm} 2$ ). Curve (a) gives the total absorption and curve (b) gives the continuum absorption.

From the absolutely normalized flashlamp spectra, one can compute an ideal singlepass pumping efficiency for Nd:glass. This is defined by assuming that all of the lamp photons pass once through the glass at normal incidence. The ideal single-pass pumping efficiency (neglecting absorption other than $\mathrm{Nd}^{3}+$ and assuming unity quantum yield) is given by:

$$
\eta_{N d}\left(P_{\text {out }}\right)=\int_{0.4 \mu \mathrm{m}}^{1.0 \mu \mathrm{m}} \mathrm{d} \lambda\left(\frac{\lambda}{1.05 \mu \mathrm{m}}\right)\left[1-\mathrm{e}^{-\beta(\lambda) \mathrm{t}}\right] \frac{\mathrm{I}\left(\lambda, \mathrm{P}_{\text {out }}\right)}{P_{\text {out }}}
$$

where an output laser photon of $1.05 \mu \mathrm{m}$ is assumed, $\beta(\lambda)$ is the $\mathrm{Nd}^{3+}$ absorption coefficient, $t$ is the disk thickness, and $I(\lambda$, Pout) is the spectrally dependent lamp intensity at that total 
output power. Notice that the designation "per unit area" is meaningless here since we define efficiency in a bulk sense so that it does not depend on the relative areas of disks and lamps. A single-pass pumping efficiency based on electrical input power is defined similarly.

Figure 5 shows the ideal single-pass pumping efficiencies based on either input power or output power predicted by our flashlamp model for phosphate laser glass at a Nddoping-thickness product of $10^{21}$ ions $/ \mathrm{cm}^{2}$. The larger values of $\eta_{\mathrm{Nd}}\left(P_{\text {out }}\right)$ compared to $\eta_{\mathrm{Nd}}\left(P_{\text {in }}\right)$ is somewhat artificial and merely reflects that the lamp output power is lower than the lamp input power for a given pumping condition. Notice that the Nd-pumping efficiency based on input power (the conventional way of defining it) is also quite high, roughly $15 \%$, at this doping-thickness product which is typical of our disk amplifiers.

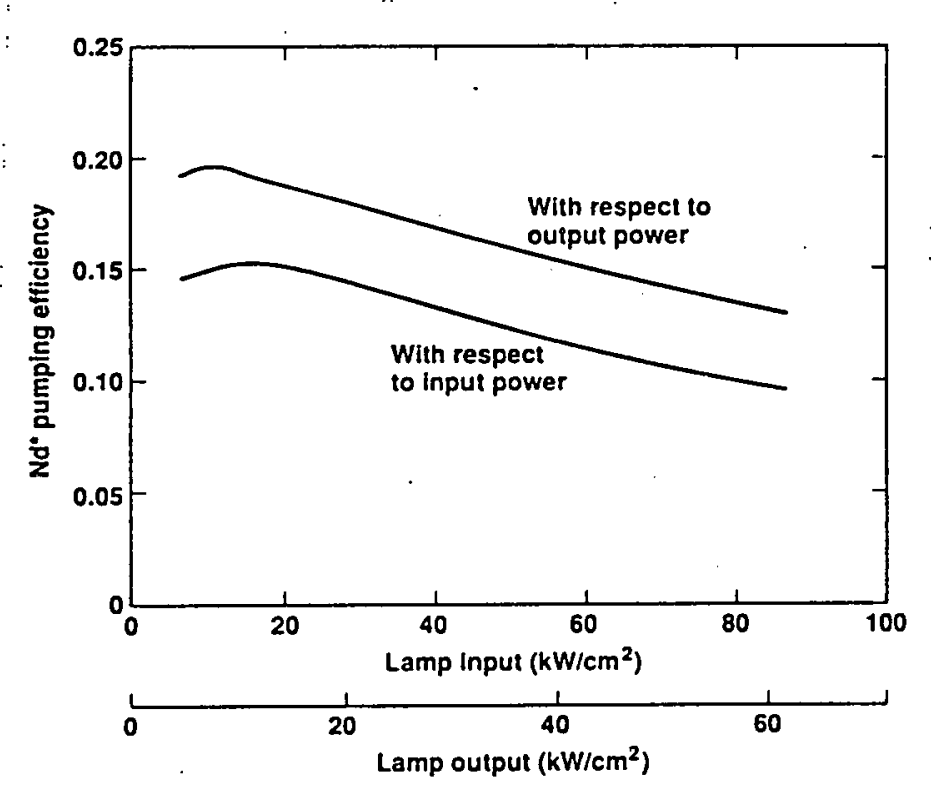

Fig. 5. Ideal single-pass pumping efficiencies versus $P_{i n}$ and $P$ out for phosphate glass at a Nd-doping-thickness product of $10^{21}$ ions $/ \mathrm{cm}^{2}$. Efficiencies were calculated with respect to output power and input power.

\section{Amplified Spontaneous Emission}

Before describing our amplifier model. it is necessary to discuss the effect of amplified spontaneous einission (ASE) inside laser disks. ASE increases the overall decay rate of excited ions and thereby decreases the energy storage efficiency. The mechanism for this is quite simple -- spontaneous emission of excited ions is amplified within the disk by other excited ions causing the latter to decay by stimulated emission. The enhancement factor of the net decay rate above the normal spontaneous rate is a function of the gain coefficient, the disk dimensions and shape, and to a small extent the profile of the gain coefficient through the thickness of the disk. Total internal reflection (TIR) has a major influence on the 
phenomenon since for disks in air approximately $75 \%\left(\sqrt{1-n^{-2}}\right)$ of the internal fluorescence is trapped inside the disk and amplified as it propagates along a zig-zag path between the disk faces. When the ASE reaches the disk edge, it can either be reflected (potentially causing parasitic oscillations), it can escape the disk, or it can be absorbed if an edge cladding is present. In the work presented here, we always used an edge cladding to minimize the effects of ASE.

Also note that TIR has the beneficial effect of decoupling disks from one another, even though they are in the same pump cavity. We have measured, but will not describe here, that the disk-to-disk ASE is indeed negligible within our amplifiers.

We measured the ASE decay rates by comparing the time dependence of $\mathrm{Nd}^{\star}$ in large and small disks. We used the set-up shown schematically in Fig. 6. A large amplifier disk (rectangular, $33 \times 65 \times 4.3 \mathrm{~cm}^{3}$ ) was mounted in our single-segment amplifier together with a small white scattering screen mounted close to the face of the amplifier disk which scattered a representative sample of the flashlamp light to a small, externally mounted diagnostic disk. Our technique requires measuring the gain in the amplifier disk and the $\mathrm{Nd}^{*}$ fluorescence from the small disk. If the external disk is small enough and pumped weakly enough, the ASE depumping rate in the small disk will be negligible. In that case its fluorescence output is proportional to the gain that the amplifier disk would produce without ASE. The ASE depumping rate can then be obtained from the differential equations for the excited states in the two disks. Details of the analysis will be given elsewhere.

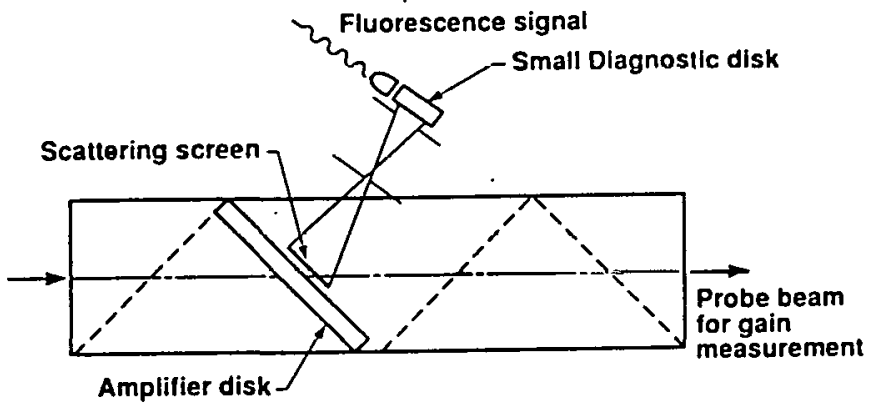

Fig. 6. Schematic diagram showing positions of the single large amplifier disk, the small diagnostic disk, and the scattering screen used for ASE measurements.

This method requires that the diagnostic disk is pumped by the same flashlamp spectrum as that which pumps the amplifier disk. For this purpose, we mounted the single amplifier disk in one of the central positions of the four-disk-long amplifier as shown in Fig. 6, where it is pumped the same on both faces. A $5-\mathrm{cm}$ square scattering screen, mounted centrally on the bottom reflector against the disk face, produced pump light for the small external disk. The angle-averaging produced by the scattering screen closely reproduces the pump spectrum seen by the disk, and a pair of apertures ensured that only light from the scattering screen reached the small disk. 


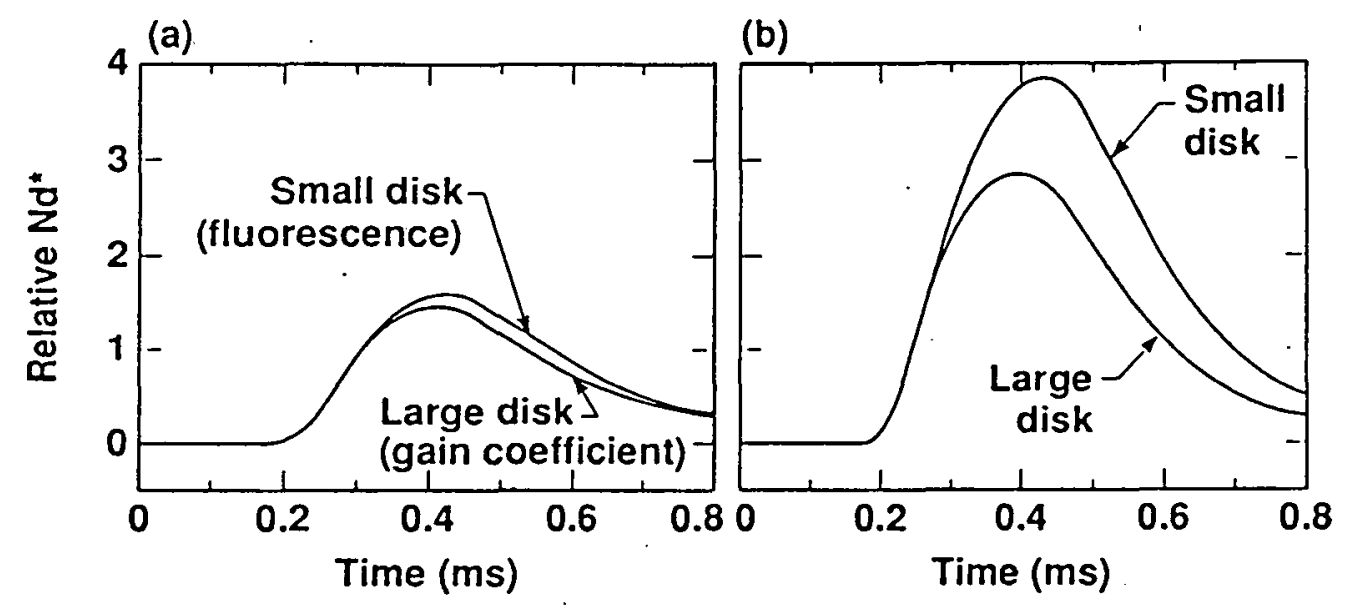

Fig. 7. Relative $\mathrm{Nd}^{\star}$ density plotted versus time for the large amplifier disk and for the small diagnostic disk. Graph (a) gives results for low flashlamp input (low ASE) and graph (b) gives results for high flashlamp input (high ASE).

Figure 7 shows signals from the two disks under low and high-input flashlamp pumping conditions. The fluorescence signal from the small disk has been scaled (using normalization from the solution of the differential equations) to show the proper magnitude of the ASE-free gain coefficient in the large disk. Low lamp loadings generate small gain coefficients and relatively small ASE effects, and there is little difference between the two. For the higher lamp loading, which is a more typical operating level, ASE reduces the largedisk gain by one-fourth and substantially shortens it in time.

Examples of the ASE decay rates inferred by this method are shown in Fig. 8. The data shown here were obtained with silver-reflective masks placed over the rectangular disk. The masks had elliptical cut-outs with long dimensions of 31,40 , and $61 \mathrm{~cm}$, which match the 15,20.8, and $31.5-\mathrm{cm}$ amplifiers of Nova: The measured ASE decay rate $\mathrm{k}_{\mathrm{ASE}}$ is plotted as a function of the instantaneous gain coefficient times the long dimension of the disk mask, gL. Notice that there is relatively good agreement in $k_{A S E}$ for times before and after the peak gain, and that appropriate scaling with the long-dimension of the mask is observed.

A model fit to the data in Fig. 8 is given by:

$$
\begin{aligned}
& k_{\text {ASE }}\left(10^{3} \mathrm{~s}^{-1}\right)=\sum_{n=1}^{5} c_{n} \cdot(g L)^{n} \\
& c_{n}=.452,-.312, .347,-.1211, .0167(n=1-5)
\end{aligned}
$$

where $g$ is the gain coefficient and $L$ is the major axis of the ellipse. The functional form of this equation was suggested by numerically-predicted ASE decay rates for the same geometry. Details of this theoretical model will be presented elsewhere. Notice that Eqn. 3 describes the ASE decay rate for an elliptical disk which gives a circular aperture when 
mounted at Brewster's angle. Numerical modeling shows that $(\mathrm{gL})$ should be multiplied by a factor of 1.12 for a rectangular disk having similar proportions to give a square aperture. 8

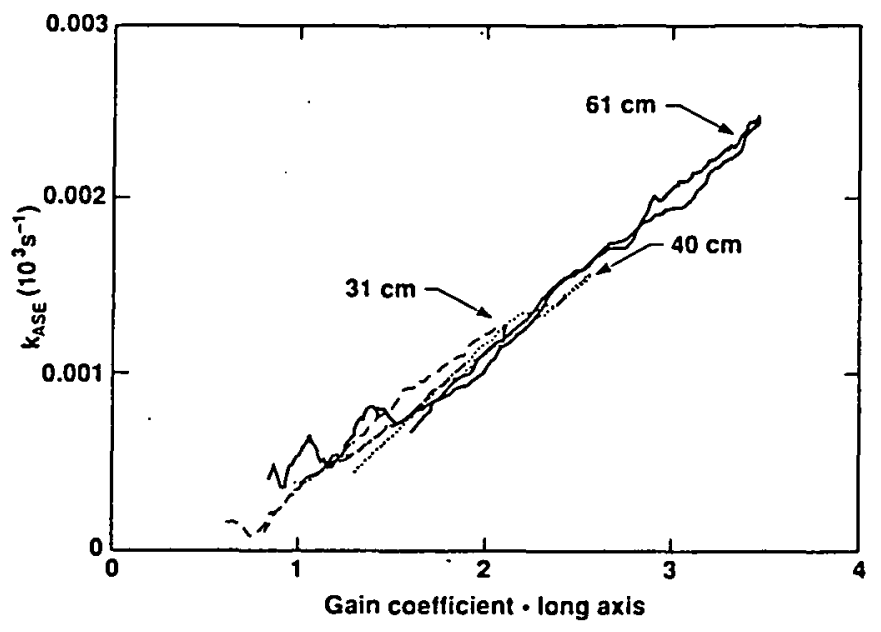

Fig. 8. Solutions for the ASE decay rates for the three aperture sizes at the maximum flashlamp loading. Each of the three experimentally derived curves is double valued since the gain coefficient first rises and then falls during each :pulse.

\section{Amplifier Pumping Model}

Combining our results of ideal single-pass flashlamp pumping and ASE decay, we can construct a differential equation to describe the energy density $\rho^{*}=N^{*}$ hv of excited $\mathrm{Nd}$ ions stored in the glass:

$$
\frac{d \rho^{\star}}{d t}-\eta_{\text {Nd }}\left(P_{\text {out }}\right) T_{\text {cav }}\left(P_{\text {out }}\right) P_{\text {out }}\left(\frac{A_{L}}{V_{d}}\right) \cdot \tau^{-1}\left(\rho^{*}\right) \rho^{*}
$$

where $P_{\text {out }}$ is the output flashlamp power per unit bore area, $T_{\text {cav }}$ is the cavity transfer efficiency from lamps to the disks, $A_{L}$ is the lamp bore area, $V_{d}$ is the disk volume, and $\tau^{-1}$ is the ASE-enhanced excited state decay rate, $k_{0}+k_{\text {ASE }}$.

The functions $\eta_{N d}\left(P_{\text {out }}\right)$ and $\tau^{-1}\left(\rho^{*}\right)$ are defined according to Eqn. (2) and Eqn. (3). The cavity transfer efficiency $T_{\text {cav }}\left(P_{\text {out }}\right)$ is a spectrally-averaged function which we determine empirically by fitting the measured amplifier gains. Notice that since it is defined relative to a single-pass pumping, the cavity transfer efficiency could be greater than unity if multiplepass pumping or optical recycling were particularly strong. Our data will show that this efficiency is generally less than unity for conditions of interest.

Since Eqn. (4) is completely parameterized in terms of the lamp output power per unit area, it is useful to have an additional equation which determines $P_{\text {out }}$ from the electrical 
input power to the lamps which is more readily measurable. As discussed in ref. 5 , the output power of clear-fused-quartz lamps is well described by the relation

$$
\frac{d P_{\text {out }}}{d t}=\left[\eta^{\prime}\left(P_{\text {out }}\right) P_{\text {in }}-P_{\text {out }}\right] / 30 \mu s
$$

where $\eta^{\prime}\left(P_{\text {out }}\right)=\left\{1-0.636 \exp \left[E(t) / 0.354 \mathrm{~J} / \mathrm{cm}^{2}\right]\right\} \eta\left(P_{\text {out }}\right)$ is an empirical modification of the instantaneous radiant efficient $\eta$ ( $\left.P_{\text {out }}\right)$ shown in Fig. 1 to account for the input energy required to establish the arc (with preionization).

We used the amplifier model specified by Eqns. 2-5 to analyze the gain data that we obtained from our single-segment amplifier (SSA) and our $2 \times 2$ multisegment amplifier (MSA).

\section{Amplifier Testbed Results}

Both the SSA and MSA employed rectangular phosphate glass (Schott LG-750) laser disks. The clear aperture of the SSA was $32 \times 32 \mathrm{~cm}$ and that of the MSA was $29 \times 29 \mathrm{~cm}$. The disks for the SSA were $4.05 \mathrm{~cm}$ thick at a Nd-doping of $4 \times 10^{20}$ ions $/ \mathrm{cm}^{3}$ while the disks for the MSA were $3.75 \mathrm{~cm}$ thick at a Nd-doping of $3 \times 10^{20}$ ions $/ \mathrm{cm}^{3}$. In both amplifiers, the disks were oriented at the Brewster's angle to the beam. The experimentally measured average $\mathrm{Nd}^{*}$ decay rates for these disks were 345 and $370 \mu \mathrm{s}$, respectively. All the disks employed the epoxy-bonded, volume-absorbing glass edge cladding which has been developed at our laboratory. 9

The pump cavity geometry for the SSA, shown schematically in Fig. 9a, represents one segment of a larger multisegment amplifier. The pump area of the four disks was contained in the vertical direction between top and bottom flat silver-plated reflectors and was pumped by either 5 or 8 flashlamps per side running in the longitudinal direction. The flashlamps had a bore diameter of $1.5 \mathrm{~cm}$ and an arc length of $234 \mathrm{~cm}$. Winston-shaped silver-plated reflectors were mounted behind the lamps in the 5-lamp case and simple cylindrical reflectors were used in the 8-lamp case. As explained previously, there is little difference in amplifier performance with different reflector shapes for a given number of lamps in the cavity.

The primary purposes of the SSA were to test the pumping efficiency with rectangular disks and to measure the performance versus lamp packing density, pump pulselength, and pulse energy. Hence, the SSA was designed for considerable flexibility.

The pump cavity for the MSA, shown schematically in Fig. 9b, represents one-fourth of a proposed $4 \times 4$ multisegment amplifier and was only one disk long. The four disks were arranged in a $2 \times 2$ matrix located between the top and bottom flat silver-plated reflectors.

The disks were pumped by 6 flashlamps in each of the two side flashlamp arrays and by 12 flashlamps in the single central flashlamp array. The lamps had a bore diameter of $2.5 \mathrm{~cm}$ and an arc length of $61 \mathrm{~cm}$. The side flashlamp arrays were similar to the arrays used in the SSA with cylindrical reflectors behind the lamps. The central array which pumps disks on both sides was fundamentally different -- it had no large curved reflectors, only small 
diamond-shaped reflectors between the lamps to reduce lamp-to-lamp transfer and to divide the radiation equally to both sides of the array. The flashlamps were oriented vertically in the MSA and were arranged to avoid direct illumination of the edges of the disk holders.
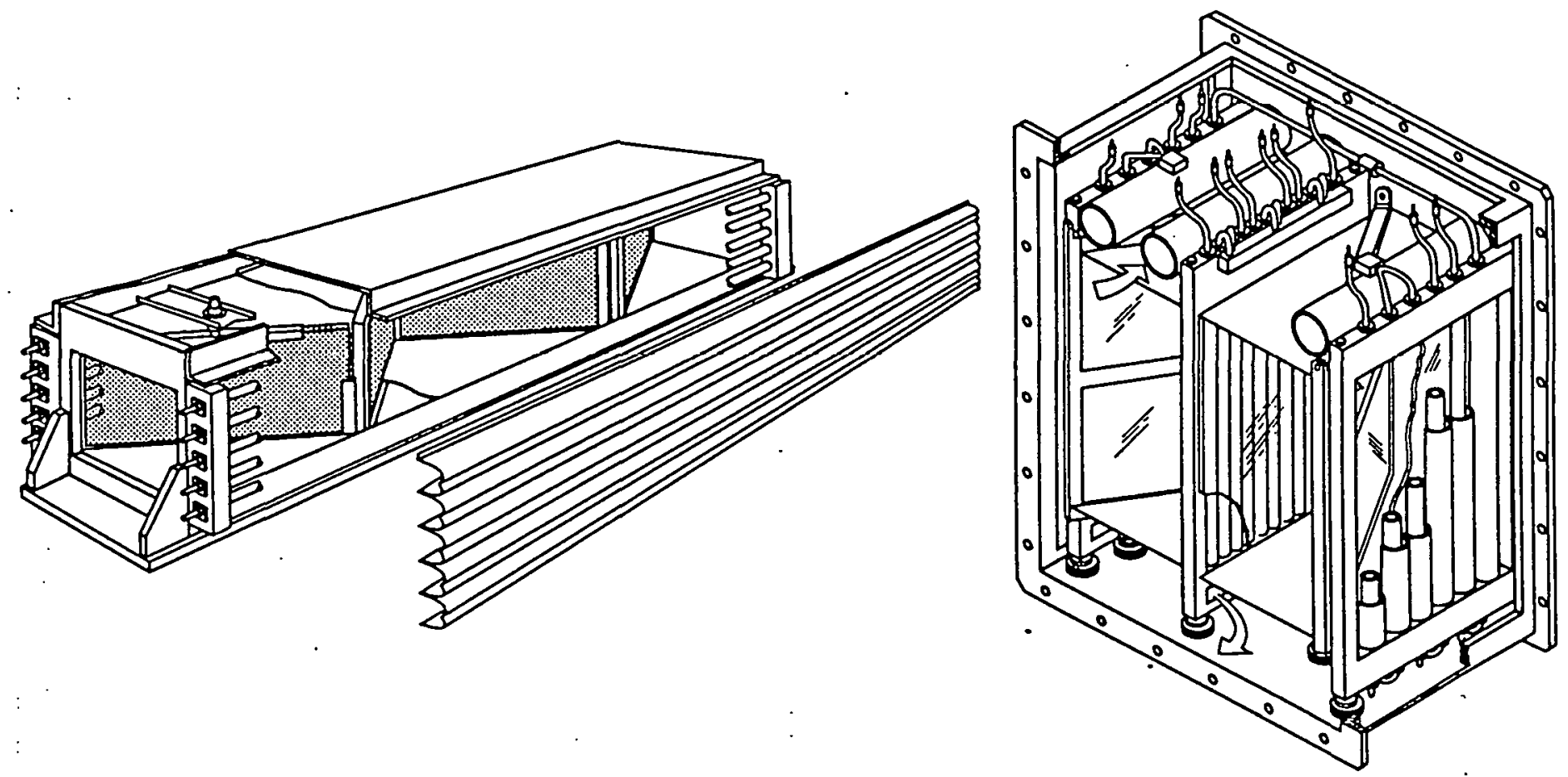

Fig. 9. Schematic diagram of the (a) 4-disk-long single-segment amplifier (SSA) and (b) the 1-disk-long multisegment amplifier (MSA).

The primary purpose of the MSA was to test the storage efficiency in this new geometry which has possible benefit from using central flashlamp arrays. Our MSA also tested the concept of single-disk-long modules, a concept that we envision using in a megajoule class device.

Since the MSA was only one disk long, the loss of pump radiation through the ends of the amplifier was significant. To determine the effect of end losses on performance and to obtain sufficient data to extrapolate our results to longer amplifiers, we made gain measurements with a flat silver-plated mirror placed over one end of the MSA to simulate an amplifier which was two disks deep. By alternating the end over which the flat reflector was placed, we simulated two-disk-long amplifiers with the disks in either a "diamond" or " $X$ " configuration. In these terms, the Brewster-angle disks form a " $V$ " shape in the MSA by itself.

We determined amplifier performance by measuring gain as a function of time using a CW Nd:YAG probe laser operating at $1.052 \mu \mathrm{m}$. We calculated stored energy $(\mathrm{hv} / \sigma \cdot \alpha)$ and hence storage efficiency from the measured peak gains using a recently measured value for the peak stimulated emission cross section of the laser glass $\left(\sigma=3.5 \times 10^{20} \mathrm{~cm}^{2}\right) .10$ Corrections were applied to account for the ditterence between the measured galn 
coefficient and the average gain coefficient over the full clear aperture of the amplifier. The experimental apparatus and techniques have been described in more detail elsewhere. 4

Figure 10 shows storage efficiency as a function of stored fluence per disk $\left(\mathrm{J} / \mathrm{cm}^{2}\right)$ for the SSA. Stored fluence per disk is defined as the extractable stored energy per unit beam area per disk. It is a useful parameter for comparing amplifiers with different apertures or amplifiers with disks of different thicknesses. Data taken for two different pump configurations at various pulselengths are plotted. The two configurations correspond to: (a) pumping with 5 lamps per side, and (b) pumping with 8 lamps per side. The pulselength values correspond to the $3 \sqrt{L C}$ value of the bank. Thus approximates the $10 \%$ to $10 \%$ width of the input power pulse for critically damped circuits.
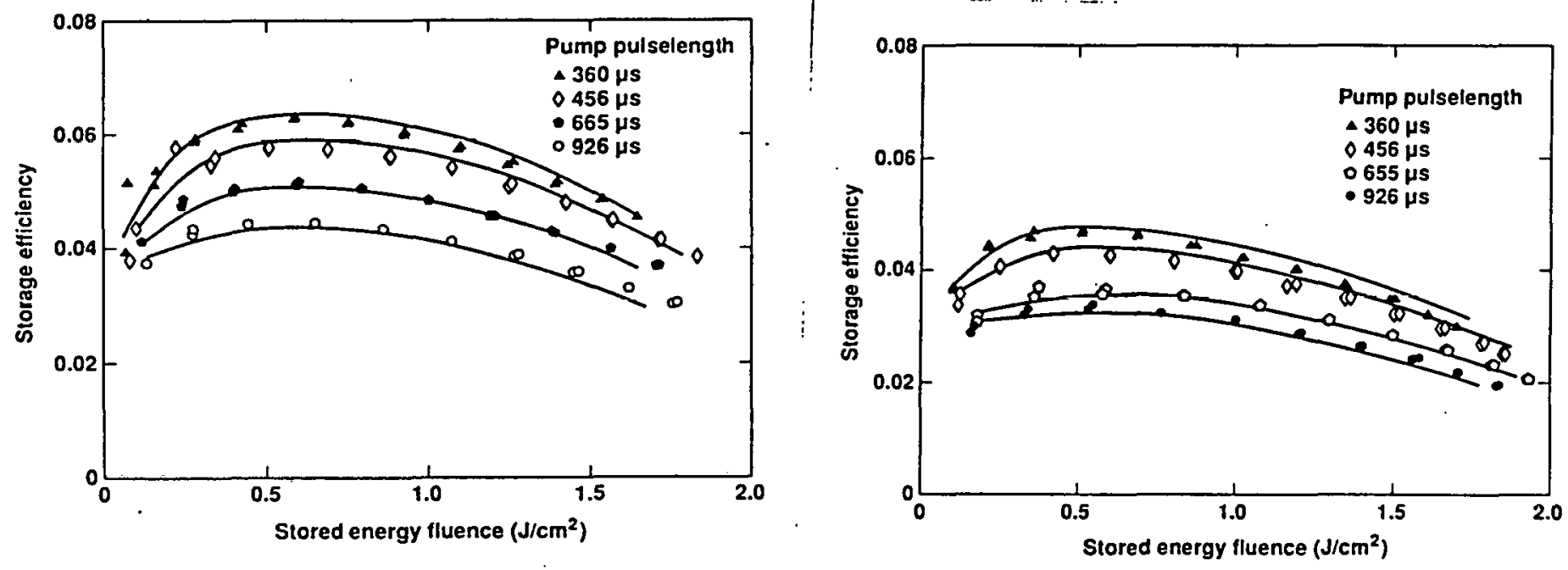

Fig. 10. Storage efficiency versus stored fluence per disk at four different pulselengths for (a) the 5-lamp per side SSA, and (b) the 8-lamp per side SSA.

The storage efficiencies of the SSA are much higher than those of our previous $\mathrm{Nd}$ :glass amplifiers. For example, when comparisons are made at comparable flashlamp pulselengths at a stored fluence per disk of $1.5 \mathrm{~J} / \mathrm{cm}^{2}$, the maximum storage efficiency of the SSA was approximately $5 \%$ compared to $2.5 \%$ for the Nova amplifiers. One reason for the improved performance of the SSA relative to the Nova amplifiers is the rectangular shape of the disks; all Nova disks are elliptical. With rectangular disks, light transported from one flashlamp array to the other must pass through a disk. Rectangular disks also increase the surface area of the disks relative to the surface area of the flashlamps and the reflectors. Another major factor for the higher cavity transfer efficiency of the SSA is the lower density of lamps (approximately one-half) compared to the Nova amplifier. Because of their high lamp densities, the large Nova amplifiers reach higher stored energy, up to $2.4 \mathrm{~J} / \mathrm{cm}^{2}$.

We determined the cavity transfer efficiency function of the SSA by simultaneously fitting the data taken at the different pulselengths. The procedure for determining the cavity transfer efficiency involved two stages. First, in order to determine the functional form giving the best fit to the data, we tried a piecewise-linear shape for the dependence of the transfer efficiency on $P_{\text {out }}$. Then to get the best fit to the measured stored energy densities, we 
varied the magnitude of the efficiency at the uniformly-spaced ."break-points". From this approach, we found that initially the efficiency decreases with increasing output and then levels off at higher loadings. This led us to choose the simple functional form

$$
T_{\text {cav }}\left(P_{\text {out }}\right)=A+B \exp \left(-C P_{\text {out }}\right)
$$

for our modeling. The parameters $A, B$, and $C$ were determined by least-squares fits to the data. The solid curves in Fig 10 are the amplifier model predictions. These predictions fit the data well and were generated using a single cavity transfer efficiency function for each configuration.

The cavity transfer efficiencies for both the 5-lamp per side and the 8-lamp per side configurations are plotted versus $P_{\text {out }}$ in Fig. 11. The shape of the cavity transfer efficiency curves can be understood by considering the amount of pump cavity light absorbed by the flashlamp plasma. At low values of $P_{\text {out }}$, where the plasma is relatively transparent, the lamps absorb little of the pump cavity radiation and the cavity transfer efficiency is high. As $P_{\text {out }}$ is increased, the plasma becomes more opaque, the lamps reabsorb more of the pump cavity radiation and the cavity transfer efficiency falls. Once $P_{\text {out }}$ becomes large enough for the plasma to be almost completely opaque (at least at the pump band wavelengths), a further increase in $\mathrm{P}_{\text {out }}$ has almost no effect on plasma opacity. In this case the cavity transfer efficiency is nearly constant.

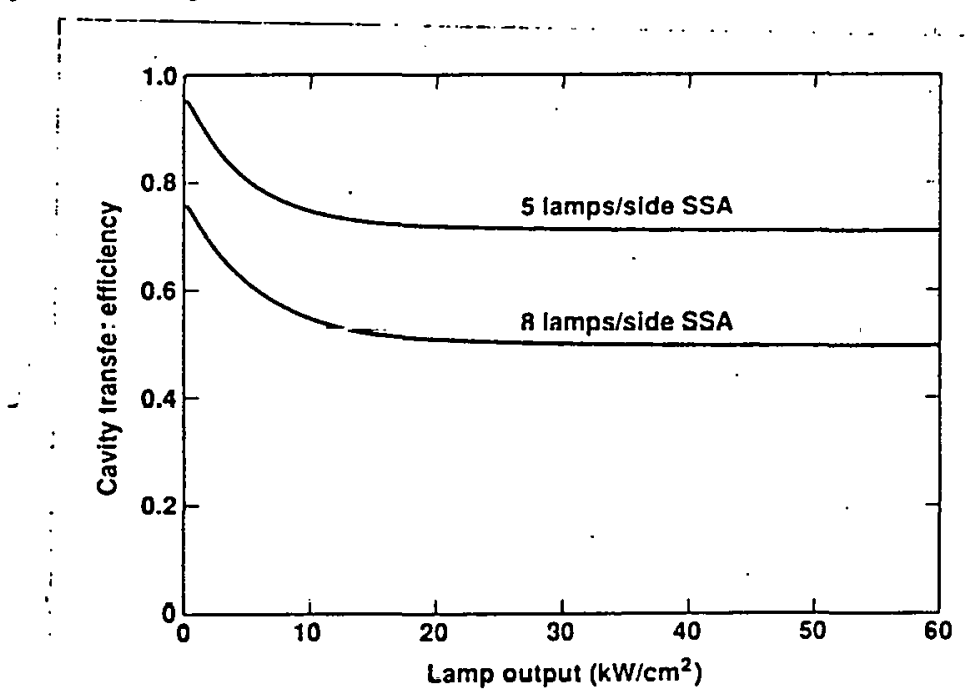

Fig. 11. Cavity transfer efficiency versus Pout for the 8-lamp per side SSA and for the 5-lamp per side SSA.

Figure 11 shows that the cavity transfer efficiency for the SSA is lower with 8-lamps per side than with 5-lamps per side. As their number is increased, the lamps absorb a larger fraction of the pump cavity radiation. This causes the cavity transfer efficiency to fall. Given the importance of the flashlamp density in determining amplifier efficiency, we have defined a new variable, the pump area ratio $(R p)$ which is the radiating wall area of the flashlamps compared to the projected wall area of the lamp panels (i.e. $\pi$ bore diameter lamp spacing). For the case of blackbody lamps, a value of unity for this parameter gives the maximum 
possible single-pass pumping. (This can be proven by conservation of brightness.) For comparison, $R_{p}$ for the $46-\mathrm{cm}$ Nova amplifier was 1.8 while for the SSA at 5 and 8 lamps per side it was 0.74 and 1.18 respectively.

The SSA results illustrate two important but unavoidable design trade-offs: increasing the lamp packing gives higher stored fluence per disk but reduces storage efficiency, and (2) driving the lamps with shorter pulses increases the storage efficiency but also decreases the stored energy when compared at the same flashlamp explosion fraction. Since these trade-offs are accurately predicted by our amplifier model, the model will be useful for cost-optimizing future large-scale ICF drivers.

Figure 12 shows storge efficiency as a function of stored fluence per disk $\left(\mathrm{J} / \mathrm{cm}{ }^{2}\right)$ for all three configurations of the MSA: the "V" configuration, the "diamond" configuration, and " $X$ " configuration. These data were taken using a $3 \sqrt{L C}$ pulse length of $500 \mu \mathrm{s}$. The MSA had a pump area ratio of 1.03 which we presume is nearly optimum. The model predictions, given by the solid curves also fit the MSA data.well.

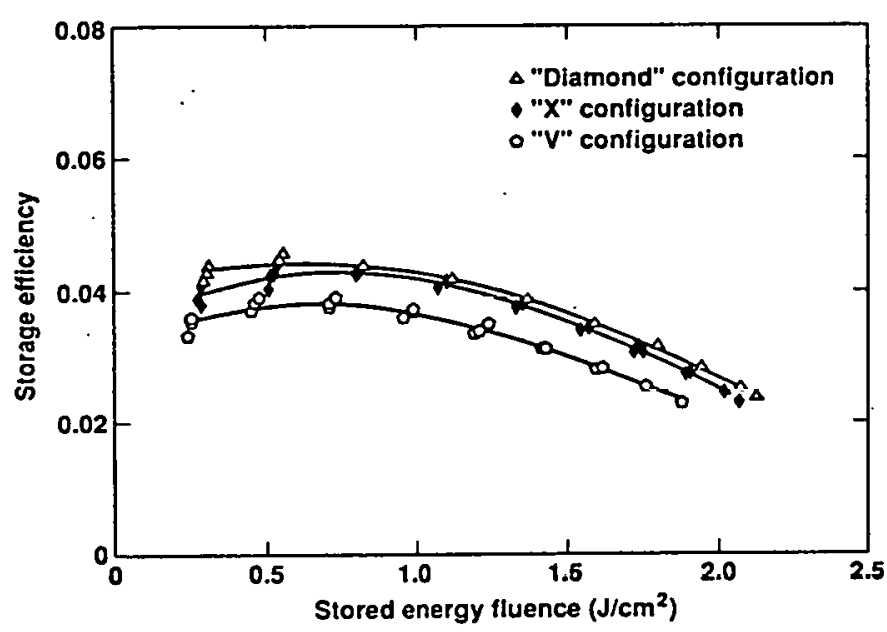

Fig. 12. Storage efficiency versus stored fluence per disk for the "diamond", "X", and " $\mathrm{V}$ " configuration of the MSA.

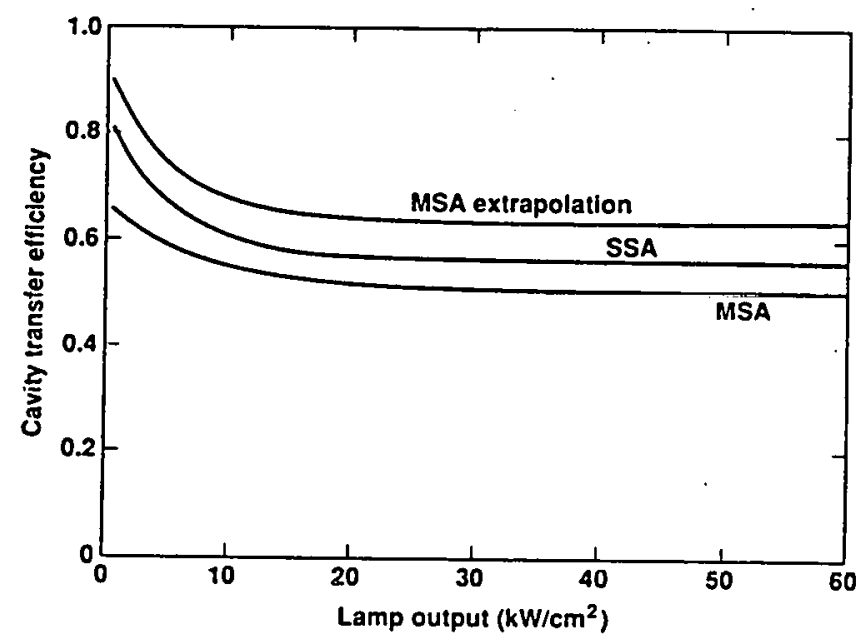

Fig. 13. Cavity transfer efficiency versus Pout for: (1) the MSA "V" amplifier; (2) the SSA with a pump area ratio equal to that of the MSA (interpolated); and (3) an MSA four-disks long (extrapolated).

The cavity transfer efficiencies of the SSA and MSA are compared in Fig. 13 The SSA curves corresponding to 5 and 8 lamps per side were interpolated to a pump area ratio of 1.0 corresponding to the MSA lamp density. Notice-that the one-disk-long case ("V" configuration) of the MSA had a slightly lower cavity transfer efficiency than the SSA, presumably because of its larger end losses. However, when we extrapolated to a fourdisk-long MSA by comparing the cavity transfer curves for the MSA with and without the silver end reflectors, we find that at comparable conditions the MSA geometry has approximately $13 \%$ higher efficiency than the SSA geometry. 
We attribute this pumping efficiency improvement mainly to the higher pumping efficiency of the central versus side lamp arrays. Other features benefitting the MSA are the transverse orientation of the flashlamps so that they are not masked by the disk holders and the fewer top and bottom reflectors per disk. We have also done other measurements (calorimetry, firing central and side lamps alone) which support the conclusion of higher pumping efficiency for the central flashlamp arrays.

\section{Conclusions and Summary}

We have developed a disk amplifier pumping model, based on experimental flashlamp and ASE data, which provides an excellent fit to measured amplifier performance. The model and the amplifier measurements both illustrate the trade-off that is made between pumping efficiency and stored energy where either the flashlamp pulselength or flashlamp packing density is varied. The model, when normalized, provides predictive capability for variations of these parameters. Hence the model should be useful for cost-optimizing largescale Nd-glass fusion drivers. We have also shown some results for the new multisegment amplifier design that we propose for these devices.

\section{Acknowledgement}

We are grateful to our many colleagues at Lawrence Livermore National Laboratory who have supported this work -- particularly to J. L. Emmett and J. B. Trenholme for inspiration and useful discussions; to F. O. Frick and E. P. Wallerstein for engineering assistance; and to B. W. Woods and R. W. McCracken for helping us assemble the hardware and take much of the data that is shown here.

\section{References}

1. J. D. Kilkenny, et. al., "Inertial Fúsion Results from Nova and Implication for the Future of ICF", 12th Intl. Conf. on Plasma Physics and Controlled Nuclear Fusion Research, IAEA, Nice, France, October 1988.

2. D. R. Speck, et. al., "Improvements in power, precision, and shot rate from the Nova target irradiation faciity", in Technical Digest, Conference on Lasers and Electro-Optics, (Optical Society of America, Baltimore, Maryland, 1989), paper TUT4.

3. W. E. Martin, J. B. Trenholme, G. J. Linford, S. M. Yarema, and C. A. Hurley, "SolidState Disk Amplifiers for Fusion-Laser Systems", IEEE J. Quantum Electron., QE-17, pp. 1744-1755 (1981).

4. J. E. Murray, H. T. Powell, and B. W. Woods, "Optimized Flashlamp Pumping of Disk Amplifiers", SPIE Proceedings Vol. 609, Conference on. Flashlamp-pumped Laser Technology, pp. 95-110, (1986).

5. H. T. Powell, A. C. Erlandson, and K. S. Jancaitis, "Characterization of High Power Flashlamps and Applications to Nd:Glass Laser Pumping", SPIE Proceedings Vol. 609, Conference on Flashlamp-pumped Laser Technology, pp. 78-94 (1986).

6. I. S. Marshak, Pulsed Light Sources (Consultants Bureau, New York, 1984). 
7. J. B. Trenholme and J. L. Emmett, "Xenon Flashlamp Model for Performance Prediction", in Proceedings of Ninth International Conference on High Speed Photography, ed. W. G. Hyzen and W. G. Chase (Society of Motion Picture and Television Engineers, New York, 1970) pp. 299-302.

8. J. B. Trenholme, "Amplifier Analysis: Fluorescence Amplification in Different Disk Geometries", 1982 Laser Program Annual Report, UCRL 50021-82, pp. 6-6 to 6-7 (1983).

9. J. H. Campbell, et. al., "Development of Composite Polymer-Glass Edge Claddings for Nova Laser Disks", in Laser-Induced Damage in Optical Materials: 1986; Proceedings of the Boulder Damage Symposium, Nov. 1986, Boulder, Colorado, pp. 19-59.

10. Private communication, J. A. Caird, Lawrence Livermore National Laboratory.

11. L. Noble, C. Kretschmer, R. Maynard, H. Flentz, and L. Reed, "Optical Pumps for Lasers", Final Report, Vol. 1, ILC Technology, Inc., Sunnyvale, CA, ECOM-0239-F, May 1973.

\section{Appendix: Description of the Flashlamp Model}

Following the precedent of Trenholme and Emmett7, we chose to describe the quasistationary output spectra of flashlamps in terms of the current density $j$ (in $\mathrm{kA} / \mathrm{cm}^{2}$ ), bore diameter $d$ (in $\mathrm{cm}$ ), and $X e$ fill pressure $p$ (in torr). As described in the text, we use the model predictions to describe non-stationary conditions when parameterized as a function of the output power per unit area. Determination of the output power requires a spectral integration of the results to be presented. It is also sometimes useful to know the electrical input power per unit area at quasi-stationary conditions as a function of $j, d$, and $p$. A good fit to our experimental results is provided by

$$
P_{\text {in }}=2.81 \exp [1.48 \ln (j)+0.90 \ln (d)+0.20 \ln (p)+0.050 \ln (j) \ln (d)]
$$

where the input power per unit area $P$ in is expressed in $\mathrm{kW} / \mathrm{cm}^{2}$. This parameterization of the electrical input power differs in magnitude and form only slightly from the $j^{1.5} p^{0.2} d$ dependence assumed in earlier analyses. 11

Using the procedure described in the text, we obtained a best-fit of the temperature of the flashlamp plasma to our experimental data with the power-law relationship

$$
T=9300 j^{0.18} d^{0.092} p^{-0.017}
$$

where $T$ is expressed in degrees Kelvin. The wavelength-dependent absorption/emission coefficient is expressed as the sum of a broad continuum curve $a_{c}(\lambda)$ and a discrete line component $a_{1}(\lambda)$, both of which are multiplied by a common scaling function $M(j, d, p)$ :

$$
\alpha(\lambda)=\left[\alpha_{c}(\lambda)+\alpha_{l}(\lambda)\right] M(j, d, p) .
$$


The magnitude factor $M$ treats the variations which depend on the flashlamp operating parameters with a modified power-law scaling of the form

$$
M(j, d, p)=\exp \left[1.57 \ln (j)+0.071 \ln ^{2}(j)+0.19 \ln (d)+0.46 \ln (p)\right]
$$
two Gaussians

Our estimate for the continuum portion of the absorption shape factor is the sum of

$$
\alpha_{c}=\alpha_{1} \exp \left\{-\left[\left(\lambda-\lambda_{1}\right) / \omega_{1}\right]^{2}\right\}+\alpha_{2} \exp \left\{-\left[\left(\lambda-\lambda_{2}\right) / \omega_{2}\right]^{2}\right\}
$$

with magnitudes $\alpha_{1}=2.37 \times 10^{-2} \mathrm{~cm}^{-1}$ and $\alpha_{2}=1.5 \times 10^{-3} \mathrm{~cm}^{-1}$, center wavelengths $\lambda_{1}=$ $0.700 \mu \mathrm{m}$ and $\lambda_{2}=0.300 \mu \mathrm{m}$, and widths $\omega_{1}=0.330 \mu \mathrm{m}$ and $\omega_{2}=0.140 \mu \mathrm{m}$. The discrete portion of the shape factor is a sum of 20 distinct Lorentzian lines whose position, height and width (FWHM) are presented in Table I-1.

Once the temperature of the plasma and the magnitude of the emission coefficient are determined, the spectral emittance of the flashlamp $I(\lambda)$ is calculated by applying Kirkhoff's law (Eqn. 1) and integrating over all output angles, with the result:

$$
I(\lambda)=I_{B B}(\lambda, T) F[\alpha(\lambda) d]
$$

In this equation $I_{B B}$ is the black-body emission at the plasma temperature, and $F$ is the angle-averaged emissivity function which is calculated using the polynomial expansions of Trenholme and Emmett (see Table 1-2)

The experimental studies from which this model were derived provided detailed information on the spectral shape only in the range from 0.4 to $1.0 \mu \mathrm{m}$. Outside of this range only the magnitude of the power emitted in the ultraviolet and infrared was measured. In the ultraviolet region $\left(\lambda<0.4 \mu \mathrm{m}\right.$ ) the emitted power per unit area of wall bore $P_{u v}$ (in $\mathrm{kW} / \mathrm{cm}^{2}$ ) is approximated by a simple power-law scaling law of the form:

$$
P_{u v}=0.174 j^{2.07} d^{1.31} p^{0.2}
$$

In the infrared region $(\lambda>1.0 \mu \mathrm{m})$ the output is described by a modified power-law:

$$
P_{i r}=0.958 \exp \left[1.11 \ln (j)+0.094 \ln ^{2}(j)+0.60 \ln (d)+0.080 \ln (p)\right]
$$

where the output power per unit area $P$ ir is expressed in $\mathrm{kW} / \mathrm{cm}^{2}$. The total radiative output per unit area Pout is the sum of these two terms and the integral of the output spectrum calculated in the range from 0.4 to $1.0 \mu \mathrm{m}$.

One can show by direct calculations that this model predicts flashlamp spectra for different bore diameters which scale closely with $\mathrm{Pin}$ and $\mathrm{Pout}$. This is by no means obvious from these formulas. 
Table I-1. Positions, magnitudes, and widths of the discrete Lorentzian lines included in the flashlamp mode.

$\begin{array}{ccc}\text { Center Wavelength }(\mathrm{nm}) & \text { Magnitude }\left(\mathrm{cm}^{-1}\right) & \text { Linewidth }(\mathrm{nm}) \\ 467.1 & 0.003 & 8 \\ 483.0 & 0.002 & 5 \\ 492.0 & 0.002 & 5 \\ 687.7 & 0.003 & 10 \\ 764.2 & 0.015 & 6 \\ 823.2 & 0.150 & 5 \\ 828.0 & 0.050 & 3 \\ 834.7 & 0.060 & 4 \\ 840.9 & 0.012 & 2 \\ 881.9 & 0.450 & 5 \\ 895.2 & 0.150 & 4 \\ 904.5 & 0.120 & 4 \\ 916.3 & 0.180 & 5 \\ 937.5 & 0.070 & 8 \\ 944.5 & 0.040 & 8 \\ 951.4 & 0.070 & 8 \\ 968.5 & 0.010 & 4 \\ 971.8 & 0.030 & 8 \\ 980.0 & 0.100 & 4 \\ 992.3 & 0.100 & 5\end{array}$

Table 1-2. Polynomial fits to the angle-averaged emissivity function (from reference 7).

$$
\text { If } x \leq 5.0 \text {, then } F(x)=\sum_{n=1}^{9} a_{n} x^{n} \text {, with }
$$

$$
\begin{array}{rlrl}
a_{1}=0.99792247 & a 6=-0.016125646 \\
a_{2}=-0.62384425 & a 7=2.7502932 \times 10^{-3} \\
a_{3}=0.33474365 & a 8=-2.6653634 \times 10^{-4} \\
a_{4}=-0.15994948 & a 9=1.1101578 \times 10^{-5} \\
a_{5}=0.060552904 & \\
\text { If } x>5.0, \text { then } F(x)=1-\sum_{n=1}^{3} c_{n}\left(\frac{1}{x}\right)^{n}, \text { with } \\
c_{1}=0.75 & \\
c_{2}=-1.875 & c_{3}=15.0
\end{array}
$$$$
a 7=2.7502932 \times 10^{-3}
$$$$
\mathrm{a} 9=1.1101578 \times 10^{-5}
$$ 
